\title{
Eğitim Amaçıı SDR Tekniklerine Dayalı FPGA Tabanlı Genlik Modüleli Radyo Vericisi Tasarımı ve Uygulaması
}

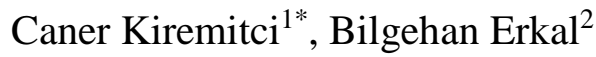 \\ ${ }^{1}$ Karabük Üniversitesi, Mühendislik Fakültesi, Elektrik Elektronik Mühendisliği Bölümü, Karabük, Türkiye (ORCID: 0000-0003-3863-4466) \\ ${ }^{2}$ Karabük Üniversitesi, Mühendislik Fakültesi, Elektrik Elektronik Mühendisliği Bölümü, Karabük, Türkiye (ORCID: 0000-0002-1405-6932)
}

( $1^{\text {st }}$ International Conference on Computer, Electrical and Electronic Sciences ICCEES 2020 - 8-10 Ekim 2020)

(DOI: 10.31590/ejosat.803492)

\begin{abstract}
ATIF/REFERENCE: Kiremitci, C. \& Erkal, B. (2020). Eğitim Amaçıı SDR Tekniklerine Dayalı FPGA Tabanlı Genlik Modüleli
\end{abstract} Radyo Vericisi Tasarımı ve Uygulaması. Avrupa Bilim ve Teknoloji Dergisi, (Özel Sayı), 184-189.

\begin{abstract}
$\ddot{O} \mathbf{z}$
Yazılım tanımlı radyo (Software Defined Radio - SDR) sistemlerinde temel amaç, radyo işaretlerini bir sayısal işaret işleyiciyle tamamen sayısal olarak işlemektir. Bu sistemler üzerinde modülasyon, demodülasyon, işaret üretimi ve hat kodlaması gibi işlemlerin alanda programlanabilir kapı dizileri gibi bir işlemci ile yapılması analog devre temelli donanıma duyulan ihtiyacı büyük ölçüde azaltmaktadır. Alanda Programlanabilir Kapı Dizileri (Field Programmable Gate Array - FPGA), programlanabilir mantık blokları arası bağlantılardan oluşan ve geniş uygulama alanları olan sayısal tümleşik devrelerdir. Tasarımcının ihtiyacına yönelik mantık işlevlerini gerçekleştirme amacıyla üretilmiştir. Bundan dolayı her mantık bloğunun işlevi kullanıcı tarafindan düzenlenebilmektedir. FPGA'in programlanması aşamasında ise genellikle VHDL (Very High Speed Integrated Circuit Hardware Description Language) kullanılır. Bu çalı̧̧ma üzerinde ilk olarak VHDL kodu yazılarak, genlik modüleli (Amplitude Modulation - AM) verici, FPGA kartı (Mimas Spartan 6) üzerinde gerçekleştirilmiştir. Audacity programı ile modülasyonda kullanılacak örnek ses kaydı, ses kartı aracılığı ile FPGA kartına gönderilmiştir. FPGA kartı, ADC (Analog / Digital Converter - LM4550) kartı üzerinden analog sinyali alarak HDSDR (High Definition Software Defined Radio) programı ile verici sinyali alınıp, demodüle edilip, kaydedilmiştir. FPGA kartı, DAC (Digital / Analog Converter - LM4550) kartı aracılı̆̆ı ile verici sinyalini analog formda üretip, laptopun ses kartı mikrofon girişine göndermiştir. Ve son olarak kaydedilmiş verici sinyali ayrıca matlab koduyla da offline olarak demodüle edilip sonuç harddiske kaydedilmiştir. Elde edilen demodüleli sinyalin baştaki modülasyon sinyaliyle aynı olduğu ve modülasyonun düzgün bir şekilde gerçekleştiği görülmüştür. Sonuç olarak, yazılım tanımlı radyo sistemlerinin (SDR), alanda programlanabilir kapı dizileri (FPGA) üzerinde gerçekleştirilmesine ve eğitimine yönelik güzel bir platform elde edilmiştir.
\end{abstract}

Anahtar Kelimeler: SDR, FPGA, VHDL.

\section{Design and Implementation of an Educational AM Transmitter with FPGA using SDR Techniques}

\begin{abstract}
In SDR systems, the main purpose is to process the radio signals completely numerically with a digital signal processor. Performing operations such as modulation, demodulation, signal generation and line coding on these systems with a processor such as FPGA greatly reduces the need for analog circuit-based hardware. FPGA are digital integrated circuits that consist of interconnections between programmable logic blocks and have wide application areas. It is produced for the purpose of realizing the logic functions needed by the designer. Therefore, the function of each logic block can be edited by the user. VHDL is generally used in programming the FPGA. In this study, firstly, VHDL code was written and AM transmitter was realized on FPGA board (Mimas Spartan 6). The sample sound recording to be used in modulation with the Audacity program was sent to the FPGA card via the sound card. FPGA card received analog signal from ADC (LM4550) card, transmitter signal was received, demodulated and recorded with HDSDR program. FPGA card generated the transmitter signal in analog form via DAC (LM4550) card and sent it to the microphone input of
\end{abstract}

${ }^{*}$ Sorumlu Yazar: Karabük Üniversitesi, Mühendislik Fakültesi, Elektrik Elektronik Mühendisliği Bölümü, Karabük, Türkiye, ORCID: 0000-00033863-4466, caner_kiremitci@ hotmail.com 
the laptop's sound card. And finally, the recorded transmitter signal was demodulated offline with the matlab code and the result was saved on the hard disk. It has been observed that the demodulated signal obtained is the same as the initial modulation signal and the modulation is performed properly. As a result, a good platform for the implementation and training of SDR systems on FPGA has been obtained.

Keywords: SDR, FPGA, VHDL.

\section{Giriş}

Yazılım tanımlı radyo (Software Defined Radio), kablosuz haberleşme amacıyla düşünülmüş ve konfigürasyonu yeniden düzenlenebilen bir donanım ve yazılım teknolojileri koleksiyonudur. Yazılım tanımlı radyoya yönelik ilk fikirler, 1991 yılında John Mittola tarafından radyoların yazılımsal olarak konfigüre edilebileceği ve programlanabileceği düşüncesiyle ortaya konulmuştur. Klasik bir donanımsal radyo sisteminde sinyal alçaltma/yükseltme, modülasyon/demodülasyon, filtreleme gibi işlemler diğer donanım elemanları üzerinden yapılır. Yazılımsal radyoda ise bu elemanların yerini kullanıcının herhangi bir zamanda değiştirebileceği, programlanabilir bir sistem bulunur. SDR sistemlerinin geliştirilmesiyle birlikte donanım tabanlı radyolara göre maliyetin düşürülmesi, işlevselliğin değiştirilebilmesi gibi avantajlar sağlamıştır. [1], [2], [3], [4], [5]

Alanda Programlanabilir Kapı Dizileri (FPGA), programlanabilir mantık blokları arası bağlantılardan oluşan ve geniş uygulama alanları olan sayısal tümleşik devrelerdir. Tasarımcının ihtiyacına yönelik mantık işlevlerini gerçekleştirme amacıyla üretilmiştir. Bundan dolayı her mantık bloğunun işlevi kullanıcı tarafından düzenlenebilmektedir. FPGA ile temel mantık bloklarının ve yapısı daha karmaşık olan devre elemanlarının işlevselliği artmaktadır. FPGA programlamak için; grafiksel tasarım ve VHDL yöntemleri kullanılır. Grafiksel tasarım, derleyici program (ISE, Quartus vs.) kütüphanesinde yer alan araçlar ve mantık kapıları kullanılarak yapılır. VHDL ise en yaygın olarak kullanılan programlama türüdür. VHDL (Very High Speed Integrated Circuit Hardware Description Language) 1980'lerden beri kullanılıp sürekli geliştirilmiş ve ayrıca IEEE tarafindan da standart olarak kabul edilmiştir. FPGA devrelerinin SDR sistemlerinde kullanılma nedenleri ise; ilk olarak, aynı anda birden fazla paralel işlem gerçekleştirebilme kapasitesine sahiptir ve yüksek sayıda giriş-çıkış ünitesi vardır.Ayrıca FPGA istenildiği gibi yeniden programlanabilir. FPGA ve SDR sistemleri birlikte kullanılarak bu özellikleriyle ile birlikte ayrıca çeşitli modülasyon yöntemlerini daha iyi alma ve iletme imkanı sağlarlar. Bu bilgiler doğrultusunda, genlik modüleli radyo vericisi, SDR teknikleri ve FPGA devresi ile birlikte VHDL kodları kullanılarak tasarlanıp uygulamaya alınacaktır. [6], [7], [8]

Bu çalışmanın amacı, yukarıda verilen bilgiler doğrultusunda, genlik modüleli radyo vericisini, SDR teknikleri ve FPGA devresi ile birlikte tasarlayıp; daha hızlı çalışan ve aynı zamanda tekrar programlanabilir olduğu için daha düşük maliyetli bir tasarım ortaya koymaktır.

\section{Materyal ve Metot}

Bu çalışmada donanımsal olarak, Mimas Spartan 6 FPGA kartı, LM4550 genişletme modülü ve IO çoğaltıcı breakout kartı kullanılmıştır. Yazılımsal olarak ise, VHDL kodlamasını gerçekleştirebilmek için ISE Design Suite 14.7, ses dosyasını düzenleme ve kaydetme için Audacity, giriş/çıkış sinyallerini kontrol edip görüntüleyebilmek için HDSDR, ve ayrıca elde edilen verici sinyalini demodüle edebilmek için de Matlab kullanılmıştır. [9], [10], [11], [12]

\subsection{Donanım Bileşenleri}

\subsubsection{Mimas Spartan 6 FPGA Kartı}

Mimas Spartan 6 FPGA kartı kullanımı kolay bir FPGA geliştirme kartıdır. (Şekil 1) Bu geliştirme kartı; Sistem tasarımını FPGA'larla denemek ve öğrenmek için uygun olacak şekilde tasarlanmıştır. Bu geliştirme kartında maksimum 70 kullanıcı IO'su ile Xilinx XC6SLX9/TQG144 FPGA'ya sahiptir. [13]

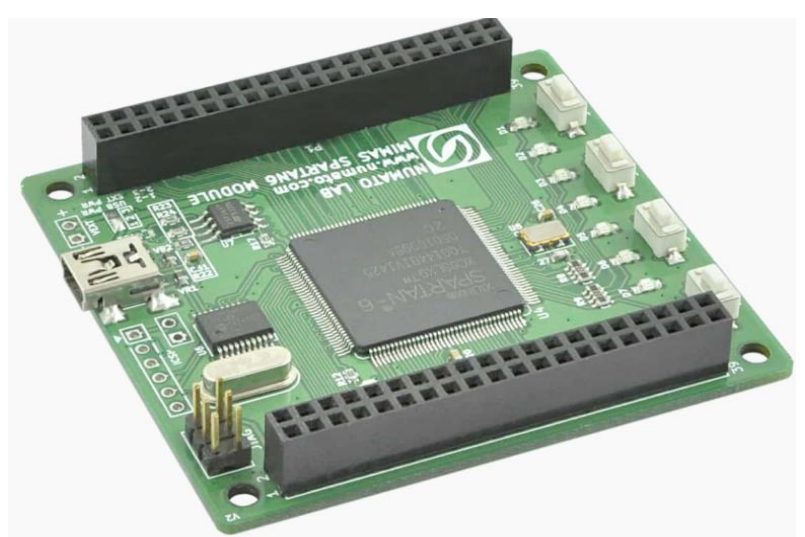

Şekil 1. Mimas Spartan 6 FPGA kartı 


\subsubsection{LM4550 Genişletme Modülüi}

LM4550 genişletme modülü, yüksek kaliteli stereo ses üretip kaydetmeyi sağlayan AC'97 Rev 2.1 uyumlu bir ses codec'i olan LM4550'ye sahiptir. (Şekil 2) Bu modül, 2×6 pin genişletme konnektörüne sahip FPGA/Mikrodenetleyici kartlarıyla kullanılmak üzere tasarlanmıştır. [13]

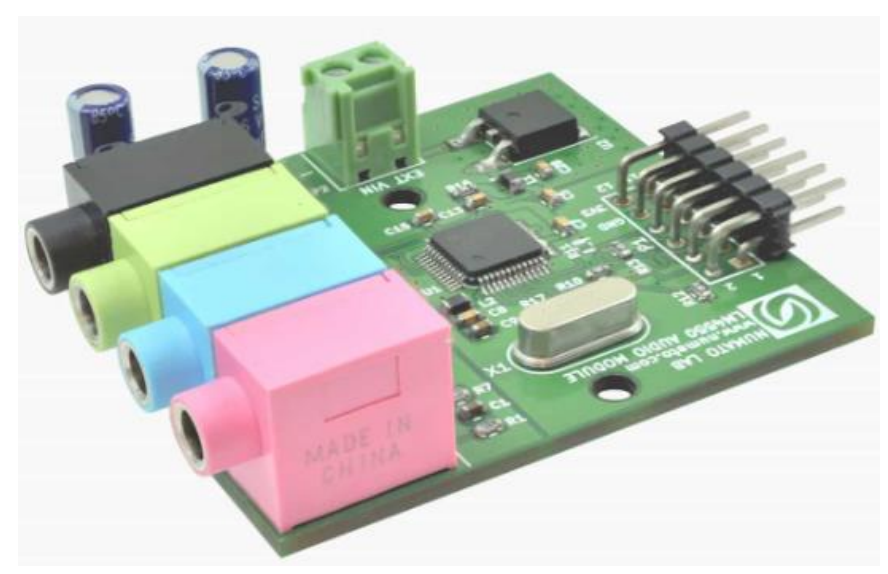

Şekil 2. LM4550 genişletme modülü

\subsubsection{IO Çoğaltıcı Breakout Kartı}

IO breakout kartı, Saturn Spartan 6 geliştirme kartı için bir IO koparma çözümüdür. (Şekil 3) Bu kart, Mimas IO'larının diğer çevresel genişletme modüllerinin kolayca takılmasını kolaylaştıracak daha küçük $2 \times 6$ başlıklara ayrılmasını sağlar. Dört adet $2 \times 6$ genişletme konektörü bulunur. [13]

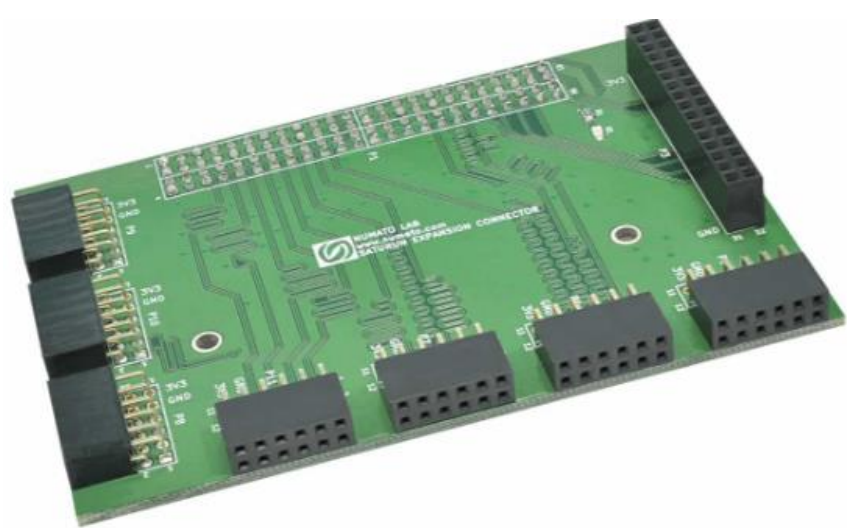

Şekil 3. IO çoğaltıcı breakout kartı

\subsection{Genlik Modülasyonu}

Genlik modülasyonu (Amplitude Modülation-AM) ilk olarak 1906 yılında Reginald Fessenden tarafından ortaya çıkarılmıştır. Genlik modülasyonunda taşıyıcı olan sinüs sinyalidir. Verici üzerinde, taşıyıcı sinus sinyalinin genliği bilgi sinyaline bağlı olarak değiştirilir. Belirtilen işlemi gerçekleştiren devreye modülatör denir. Alıcı üzerinde bu işlemin tam tersi olarak genlik değişikliği bilgi sinyaline çevrilir. Alıcı üzerinde gerçekleştirilen bu işleme genlik modülasyonu, gerçekleştiren devreye de demodulator denir.

Genlik modülasyonunda bilgi sinyalinin genlik ve frekansına göre taşıyıcı sinyalin genliği değiştirilir. Uzak noktalara gönderilmek istenen düşük frekanslı bilgiler ilk olarak elektrik enerjisine çevrilir sonrasında taşıyıı sinyal üzerinden elektromanyetik dalgalar olaral uzak noktalara ulaştırılır. Alçak frekans bilgi sinyalinin pozitif alternansında taşıyıcının genliği artmaktadır. En büyük genlik ise bilgi sinyalinin tepe noktasında görülmektedir. Alçak frekans bilgi sinyalinin negatif alternansında ise taşıyıcının genliği azalmaktadır.. En küçük genlik ise bilgi sinyalinin eksi tepe noktasında görülmektedir. Burada modüle edilen sinyal bilgi sinyali, module eden sinyal ise taşıyıcı sinyalidir. Elde edilen sinyale ise modüleli sinyal denir. [14], [15] 


\subsection{Radyo Vericisinin VHDL Kodları ile Tasarımı}

ISE Design Suite üzerinden ilk başta yeni bir proje oluşturulup, kullanılan FPGA kartının modeline göre gerekli seçimler yapılmıştır. (Şekil 4)

\begin{tabular}{|l|l|}
\hline Project File: & amtx.xise \\
\hline Module Name: & amtx \\
\hline Target Device: & xc6s|x9-3tag144 \\
\hline Product Version: & ISE 14.7 \\
\hline Design Goal: & Balanced \\
\hline Design Strategy: & Xlinx Default (unlocked) \\
\hline Environment: & System Settings \\
\hline
\end{tabular}

Şekil 4. Projeyi oluşturma

Kullanılan bileşenlerin pinlerine göre input/output verilip kodlamaları gerçekleştirilmiştir. (Şekil 5) Pinlemelerdeki input / output değerlendirilmesi bileşenlerin dökümanlarında gösterilen değerlere uygun olarak verilmiştir. (Şekil 6) [16]

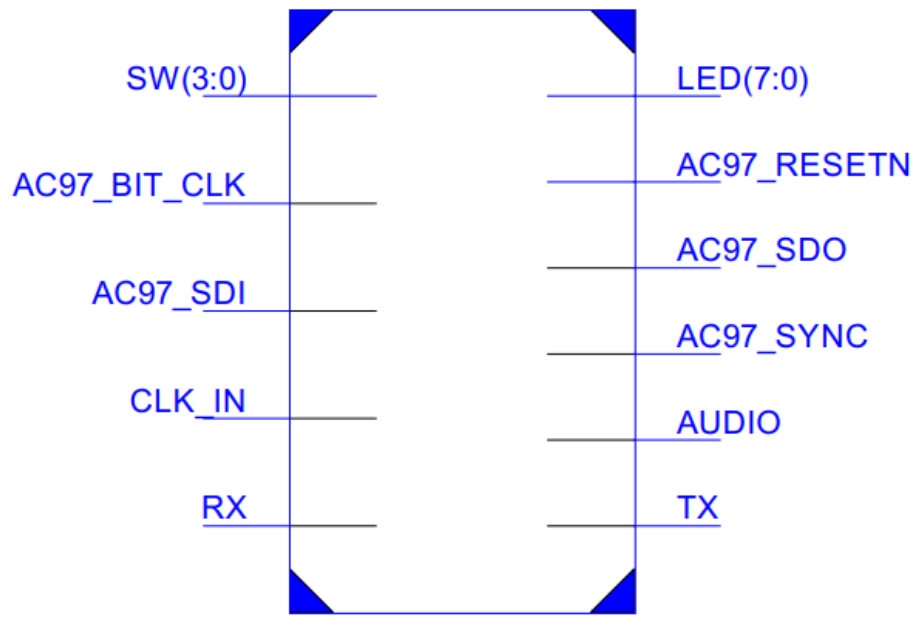

Şekil 5. Input/output şeması

\begin{tabular}{|l|l|l|l|l|}
\hline P16 & AC97_RESE... & IOB & IO_L43N_GCLK22_IRDY2_3 & OUTPUT \\
\hline P17 & AC97_SYNC & IOB & IO_L43P_GCLK23_3 & OUTPUT \\
\hline P18 & & & VCCO_3 & \\
\hline P19 & & & VCCINT & \\
\hline P20 & & & VCCAUX & \\
\hline P21 & AC97_SDI & IOB & IO_L42N_GCLK24_3 & INPUT \\
\hline P22 & AC97_BIT_... & IOB & IO_L42P_GCLK25_TRDY233 & INPUT \\
\hline P23 & & IOBS & IO_L41N_GCLK26_3 & UNUSED \\
\hline P24 & AC97_SDO & IOB & IO_L41P_GCLK27_3 & OUTPUT \\
\hline
\end{tabular}

Şekil 6. Input/output VHDL şematiği 
Mimas Spartan 6 FPGA kartına uygun olarak, oluşturulacak radyo vericisi için, LM4550 ve IO breakout kartları, PL2303 seri kabloları ve harici ses kartı ile birlikte bir devre oluşturulmuştur. (Şekil 7)

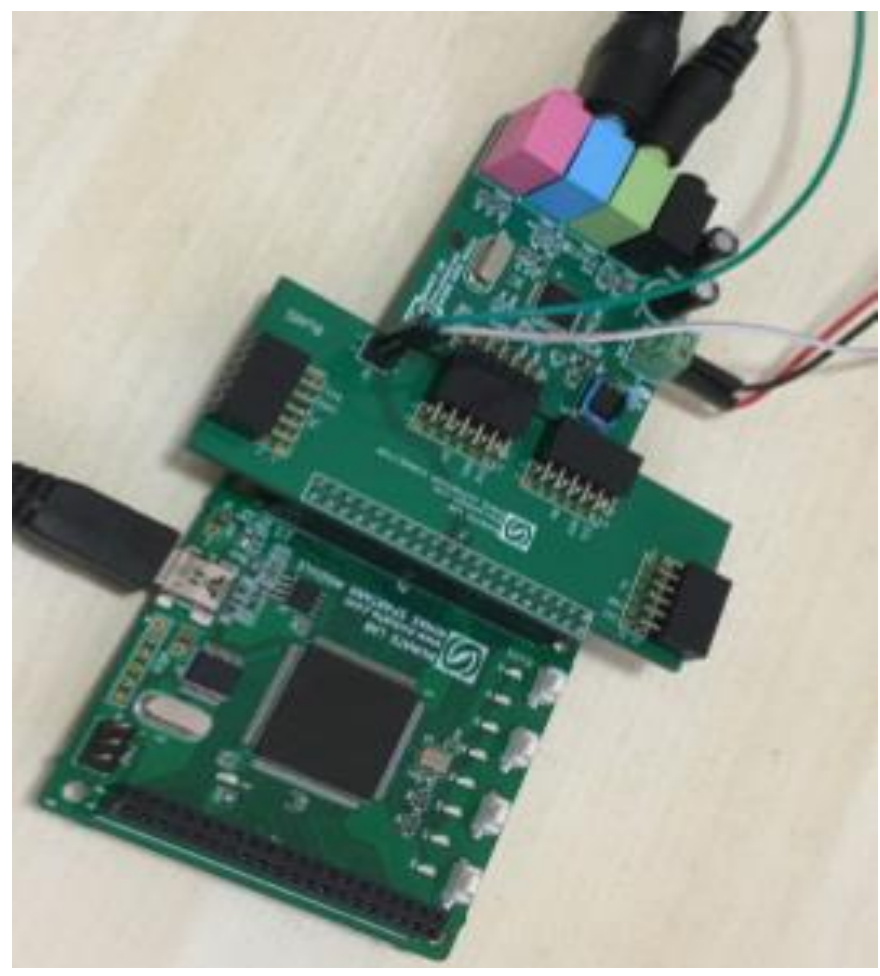

Şekil 7. Radyo vericisi devresi

AM verici, oluşturulan devre üzerinden, yazılan VHDL kodlar ile birlikte gerçekleştirilmiştir. Devre üzerinde audacity programından faydalanılarak modülasyonda kullanılacak örnek ses kaydı, ses kartı aracılı̆̆ı ile Mimas Spartan 6 FPGA kartına gönderilmiştir. FPGA kartı, LM4550 kartı üzerinden analog sinyali alarak HDSDR programı ile verici sinyali alınıp, demodüle edilip, kaydedilmiştir. FPGA kartı ise LM4550 kartı aracılığı ile verici sinyalini analog formda üretip, laptopun ses kartı mikrofon girişine göndermiştir ve son olarak elde edilen sonuçlar incelenmiştir. (Şekil 8)

\begin{tabular}{|l|l|}
\hline Objects & \\
\hline Simulation Objects for amtx \\
\hline In
\end{tabular}
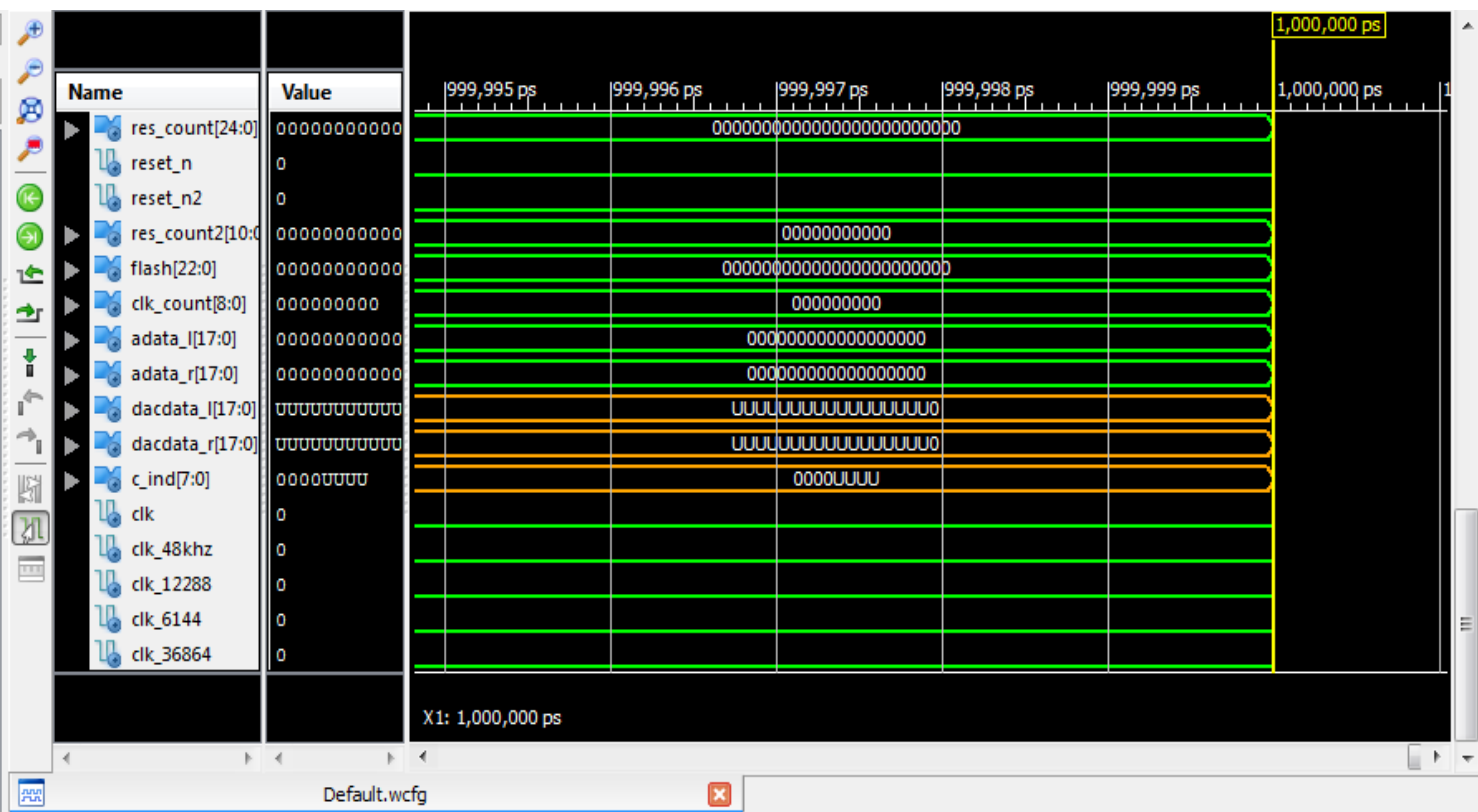

Default.wcfg

Şekil 8. ISE Design Suite simülasyonu 


\section{Araştırma Sonuçları ve Tartışma}

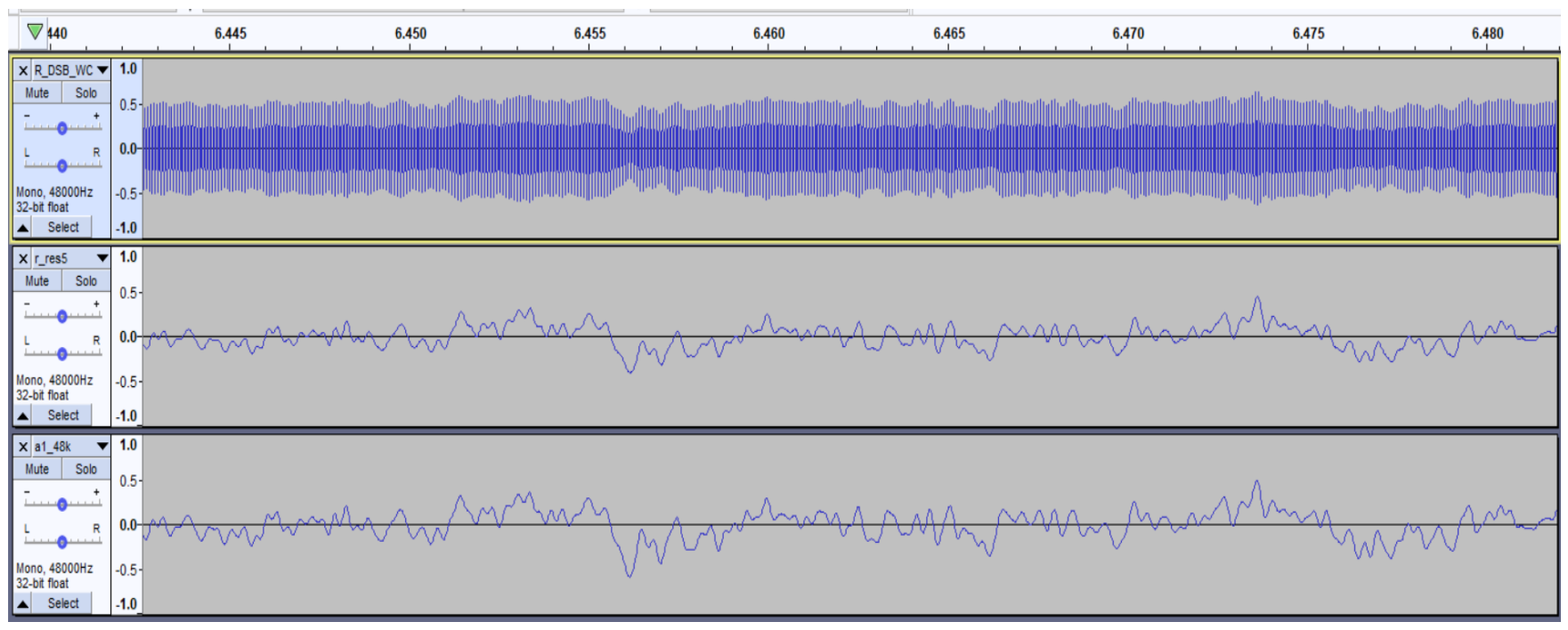

Şekil 9. Sinyallerin audacity ile görüntülenmesi

Oluşturulan bu tasarımda elde edilen sinyaller HDSDR ile kaydedilip, audacity ile görüntülenmiş ve sonucunda dinleme gerçekleştirilmiştir. Şekil 9' da gösterilen sinyaller sırasıyla en üstten en alta doğru; modüleli sinyal, demodülasyon sonucunda elde dilen sinyal ve orjinal module edici sinyaldir.

Yine şekil 9‘da gözlemlendiği üzere demodülasyon sonucu ile ilk başta modülasyon esnasında elde edilen dalgalar arasında hiçbir fark gözlenmemektedir. Dolayısıyla modülasyonun yüksek kalite ile gerçekleştirildiği görülmektedir.

\section{Sonuç}

Sonuç olarak elde edilen demodüleli sinyalin baştaki modülasyon sinyaliyle aynı olduğu ve modülasyonun düzgün bir şekilde gerçekleştiği görülmüştür. Elde edilen sonuçtan yola çıkarak SDR sistemlerinin FPGA üzerinde gerçekleştirilmesine ve eğitimine yönelik güzel bir platform elde edilmiştir.

\section{Kaynakça}

[1] Collins T.F., Getz R., Pu D. \& Wyglinski A.M. (2018) Software Defined Radio for Engineers,

[2] Mady Z.G.A. (2016). Transmit and Receive of Quadrature Phase-Shift Keying (QPSK) Signal Using Softrock SDR and Matlab Natural and Appliance Science of Karabuk University, Turkey

[3] Gareane A.G.A (2016). Transmit and Receive of FM Signals Using Softrock SDR and Matlab, Natural and Appliance Science of Karabuk University, Turkey

[4] Eame M.A.M (2016). Transmit and Receive of FSK Signals Using Softrock SDR and Matlab, Natural and Appliance Science of Karabuk University, Turkey

[5] Feng Z. (2013). A Software Defined Radio Implementation Using Matlab, Vaasan Ammattikorkeakoulu University of Applied Sciences, Finland

[6] Kara F. (2015). VHDL Kullanarak OFDM Gerçeklenmesi, Bülent Ecevit Üniversitesi Fen Bilimleri Enstitüsü, Türkiye.

[7] Akpolat A.N. (2015) FPGA Tabanlı Nesne Algılama, Firat Üniversitesi Fen Bilimleri Enstitüsü, Türkiye

[8] Cai X, Zhouand M. \&Huang X. (2017, April), "Model Based Design for Software Defined Radio on an FPGA,” IEEE Access, April 2017.

[9] Mathworks Systems (2020). Erişim Adresi: https://www.mathworks.com/products/matlab.html

[10] Audacity Audio Software Systems (2020) Erişim Adresi: https://www.audacityteam.org

[11] High Definition Software Defined Radio (2020) Erişim Adresi: http://www.hdsdr.de/

[12] ISE Design Suite (2020) Erişim Adresi: https://www.xilinx.com/products/design-tools/ise-design-suite.html

[13] Numato Systems (2020) Erişim Adresi: http://numato.com/

[14] Kibar A.E. (2019). Genlik Modülasyonu Kullanarak Güç Sinyallerinde Harmonik Çözümleme, Gazi Üniversitesi Fen Bilimleri Enstitüsü, Türkiye

[15] Alghiryani S.G.S. (2016). Transmit and Receive of SSB and DSB-AM Signals Using Softrock SDR and Matlab, Natural and Appliance Science of Karabuk University, Turkey

[16] M.F. (2012). Introduction to Spartan 3E FPGA and VHDL 